\title{
Observing the Effects of Galaxy Interactions on the Circumgalactic Medium
}

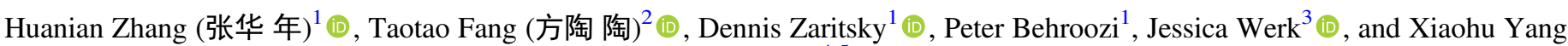 \\ (杨小 虎 $)^{4,5}$ (1) \\ ${ }^{1}$ Steward Observatory, University of Arizona, Tucson, AZ 85719, USA; fantasyzhn@email.arizona.edu \\ ${ }^{2}$ Department of Astronomy, Xiamen University, Xiamen, Fujian, People's Republic of China \\ ${ }^{3}$ Department of Astronomy, University of Washington, Seattle, WA 98195, USA \\ ${ }^{4}$ Department of Astronomy, School of Physics and Astronomy, Shanghai JiaoTong University, Shanghai, 200240, People's Republic of China \\ ${ }^{5}$ Tsung-Dao Lee Institute, and Shanghai Key Laboratory for Particle Physics and Cosmology, Shanghai Jiao Tong University Shanghai, 200240, People's Republic of \\ China \\ Received 2019 December 6; revised 2020 March 12; accepted 2020 March 16; published 2020 April 7
}

\begin{abstract}
We continue our empirical study of the emission line flux originating in the cool $\left(T \sim 10^{4} \mathrm{~K}\right)$ gas that populates the halos of galaxies and their environments. Specifically, we present results obtained for a sample of galaxy pairs with a range of projected separations, $10<S_{p} / \mathrm{kpc}<200$, and mass ratios $<1: 5$, intersected by 5443 Sloan Digital Sky Survey lines of sight at projected radii of $10-50 \mathrm{kpc}$ from either or both of the two galaxies. We find significant enhancement in $\mathrm{H} \alpha$ emission and a moderate enhancement in [N II]6583 emission for low-mass pairs (mean stellar mass per galaxy, $\bar{M}_{*},<10^{10.4} M_{\odot}$ ) relative to the results from a control sample. This enhanced H $\alpha$ emission comes almost entirely from sight lines located between the galaxies, consistent with a short-term, interaction-driven origin for the enhancement. We find no enhancement in $\mathrm{H} \alpha$ emission, but significant enhancement in [N II]6583 emission for high-mass $\left(\bar{M}_{*}>10^{10.4} M_{\odot}\right)$ pairs. Furthermore, we find a dependence of the emission line properties on the galaxy pair mass ratio such that those with a mass ratio below 1:2.5 have enhanced [N II]6583 and those with a mass ratio between 1:2.5 and 1:5 do not. In all cases, departures from the control sample are only detected for close pairs $\left(S_{p}<100 \mathrm{kpc}\right)$. Attributing an elevated $[\mathrm{N}$ II $] 6583 / \mathrm{H} \alpha$ ratio to shocks, we infer that shocks play a role in determining the circumgalactic medium properties for close pairs that are among the more massive and have mass ratios closer to $1: 1$.
\end{abstract}

Unified Astronomy Thesaurus concepts: Circumgalactic medium (1879); Galaxy pairs (610); Interacting galaxies (802)

\section{Introduction}

Even before we developed an understanding of the central role that galaxy interactions, mergers, and accretion events play in the current picture of hierarchical galaxy evolution, such phenomena were a major area of study (e.g., Toomre \& Toomre 1972). A large set of literature now addresses how these events affect the central galaxy, including evidence for large inflows of gas into the centers of galaxies (e.g., Barnes \& Hernquist 1996; Rampazzo et al. 2005) or active galactic nuclei (e.g., Springel et al. 2005; Hopkins \& Hernquist 2006), mixing of gas as evidenced by the change of behavior in metallicity gradients (e.g., Kewley et al. 2006, 2010), the enhancement of star formation and deficit in gas phase metallicity in close galaxy pairs (Ellison et al. 2013), and the prevalence of interaction signatures among post-starburst galaxies (Zabludoff et al. 1996). If dynamical effects have measurable effects on the central galaxy, then they must have even larger effects on matter at the periphery.

Galaxies are surrounded by extended and diffuse gas, referred to as the circumgalactic medium (CGM), which is a critical but incompletely understood part of galactic ecosystems. The CGM provides fuel for subsequent star formation activities (Spitzer 1956) and serves as the dumping ground for galactic recycling and feedback (Tumlinson et al. 2017). Although the existence of this component was established from the study of absorption lines in the spectra of bright background objects (Bahcall \& Spitzer 1969; Boksenberg \& Sargent 1978; Tytler 1982) and continues to be an active field of research (e.g., Steidel et al. 2010; Bordoloi et al. 2011;
Ménard et al. 2011; Zhu \& Ménard 2013a, 2013b; Werk et al. 2014, 2016; Croft et al. 2016, 2018; Cai et al. 2017; Chen 2017a, 2017b; Prochaska et al. 2017; Zahedy et al. 2017; Johnson et al. 2018; Joshi et al. 2018; Lan \& Mo 2018; Chen et al. 2019; Zahedy et al. 2019), the nature of those studies, with a single line of sight through the halo of a modest number of galaxies, makes it difficult to explore the CGM properties in subclasses of galaxies. Here, specifically, we are interested in exploring how the CGM might be affected by galaxy-galaxy interactions. Simulations indeed predict that major mergers may have a dramatic effect on the metal content and ionization state of the gas in the CGM (Hani et al. 2018).

With the advent of the detection of optical emission lines from the CGM of normal, low-redshift galaxies (Zhang et al. 2016), we now have a way to build up measurements of the CGM in specific subsamples of galaxies and constrain the CGM properties as a function of many different variables. By suitably selecting subsamples drawn from the over 7 million lines of sight from the Sloan Digital Sky Survey Data Release 12 (SDSS DR12; Alam et al. 2015), we have characterized the nature of the emission lines and the CGM in low-redshift galaxies and their environments (Zhang et al. 2016, 2018a, 2018b, 2019, 2020). We will now simply examine the corresponding emission line properties of lines of sight projected near close galaxy pairs. We adopt an imprecise $\Lambda$ CDM cosmology with parameters $\Omega_{m}=0.3$, $\Omega_{\Lambda}=0.7, \Omega_{k}=0$ and the dimensionless Hubble constant $h=0.7$ (see Riess et al. 2018; Planck Collaboration et al. 2018). 


\section{The Data}

We begin by describing the set of paired galaxies about which we measure the emission flux from the cool CGM. We identify pairs using the galaxy positions and redshfits from the SDSS Data Release 7 (DR7; Abazajian et al. 2009) and extract measures of each galaxy's Sérsic index $(n)$ and absolute magnitude $(M)$ from Simard et al. (2011), stellar mass $\left(M_{*}\right)$ from Kauffmann et al. (2003a, 2003b) and Gallazzi et al. (2005), and current star formation rate (SFR) from Brinchmann et al. (2004). For what we call our "main" sample, we require the galaxy pair projected separation, $S_{p}$, between the two galaxies, calculated from the average angular diameter distance of the two galaxies, to be greater than $10 \mathrm{kpc}$ and less than $200 \mathrm{kpc}$, the velocity offset, $\Delta V$, to be less than $1000 \mathrm{~km} \mathrm{~s}^{-1}$, and the mass ratio of the two galaxies to be no greater than 1:5 (to be clear, closer to $1: 1$ is allowed). In total, we find 52,721 galaxy pairs. Because we are interested in measuring the effect of the close interaction on the CGM properties, we also construct a "control" sample of galaxy pairs for which $400<S_{p} / \mathrm{kpc}<600,|\Delta V|<1000 \mathrm{~km} \mathrm{~s}^{-1}$ and the mass ratio less than 1:5. The control sample contains 75,007 pairs. We will also discuss the effect on our results of adopting a smaller velocity difference criterion of $500 \mathrm{~km} \mathrm{~s}^{-1}$ below, but unless otherwise noted our discussion is based on the original criteria. To address the possibility that one galaxy may pair with different galaxies at different $S_{p}$, we keep only the pairing with the smallest $S_{p}$. This choice is informed by our hypothesis that it is the closer pairings that have the greatest effect on the CGM. Of course, the smallest $S_{p}$ does not guarantee the smallest real separation, but this is the best we can do.

According both to previous theoretical studies (e.g., Kereš et al. 2005) and the empirical study of the CGM emission line ratios (Zhang et al. 2018b), there is a qualitative change in the nature of the CGM for galaxies above and below a stellar mass of $\sim 10^{10.4} M_{\odot}$. Therefore, we divide our sample into a lowmass subsample with $9.5<\log \left(\bar{M}_{*} / M_{\odot}\right) \leqslant 10.4$ and a highmass subsample with $10.4<\log \left(\bar{M}_{*} / M_{\odot}\right) \leqslant 11.5$, where $\bar{M}_{*}$ is the average stellar mass of the galaxies in the pair system.

We collect the spectra for lines of sight projected within $50 \mathrm{kpc}$ of either galaxy of each galaxy pair from the SDSS DR12. There are 5,443 such lines of sight for the main sample and 10,427 for total for control sample. For each such spectrum, we fit and subtract a tenth-order polynomial to a $300 \AA$ wide section surrounding the wavelength of $\mathrm{H} \alpha$ at the related galaxy redshift to remove the continuum. We then measure the residual $\mathrm{H} \alpha$ and $[\mathrm{N} \mathrm{II}] 6583$ flux within a velocity window corresponding to $\pm 275 \mathrm{~km} \mathrm{~s}^{-1}$ from the related galaxy to capture the majority of the emission flux from the gas surrounding that galaxy. Of the two [N II] emission lines near $\mathrm{H} \alpha$, we measure only [ $\mathrm{NII} 6583$ because, as we have determined previously (Zhang et al. 2016, 2018a, 2018b), [N II]6548 is far weaker and we have trouble measuring it in our stacks. Hereafter, we refer to [N II]6583 simply as [N II]. This procedure replicates what we have done previously (Zhang et al. 2016) and various tests and controls are discussed in our previous studies. We will also explore the sensitivity of our results to opening up the velocity window to $\pm 450 \mathrm{~km} \mathrm{~s}^{-1}$.

\section{Results}

We present stacked measurements of the $\mathrm{H} \alpha$ and [N II] fluxes for projected radii between 10 and $50 \mathrm{kpc}$ from each of the galaxies as a function of projected pair separation for each of the two mass-selected subsamples. The results ${ }^{6}$ are presented in Table 1. In figures and tables we present the median emission line fluxes and the associated uncertainties, estimated using a jackknife method. Specifically, we randomly select half of the individual spectra, calculate the mean emission line flux, and repeat the process 1000 times to establish the distribution of measurements from which we quote the values corresponding to the 16.5 and 83.5 percentiles as the lower and upper uncertainties, respectively. We only present results for $\mathrm{H} \alpha$ and [N II] although we attempted to measure the flux for [O II] 3727,3729 and [O III]5007. We did not obtain measurements that were significantly greater than zero for any of the oxygen lines.

\subsection{Emission Line Fluxes and Mean Stellar Mass}

In Figure 1 we present the $\mathrm{H} \alpha$ and [N II] emission fluxes as a function of the projected separation between the two galaxies in the pair, divided into two subsamples based on the mean stellar mass of the galaxy pair. Among the lower-mass pairs, we find a significant $(>4.7 \sigma)$ enhancement of the $\mathrm{H} \alpha$ flux over the control value at the smallest pair separations and consistency with the control sample at greater separations. Among the higher-mass pairs, the $\mathrm{H} \alpha$ flux is not significantly enhanced at any pair separation. Instead, [N II] is elevated in pairs with small and intermediate separations. Combining the inner two radial bins, the enhancement in [N II] is significant at the $97.5 \%$ $(2.5 \sigma)$ confidence level. To determine the sensitivity of our measurements on our definition of pairs, we decreased the velocity difference criterion for pair identification to $500 \mathrm{~km} \mathrm{~s}^{-1}$ and find only modest $(<10 \%)$ flux differences.

Given the nature of galaxy interactions, and the possibility of high-velocity gas inflows or outflows, we now examine the sensitivity of our results to our defined spectral velocity window. We expand the window to $\pm 450 \mathrm{~km} \mathrm{~s}^{-1}$, a compromise between expanding the window as wide as possible and minimizing the introduction of additional noise and contamination. We find a diminution of the $\mathrm{H} \alpha$ signal for low-mass pairs at the smallest $S_{p}$, from $0.033 \pm 0.006$ to $0.017 \pm 0.005$ (a $2 \sigma$ difference), which is still consistent with a significant flux enhancement but suggestive that there is no additional large flux contribution from high-velocity gas that we are missing in our original measurement. The differences obtained in $\mathrm{H} \alpha$ fluxes for either the next $S_{p}$ bin and for the control bin are statistically insignificant, as are the results for the [N II] detection in the high-mass pair subsample. We conclude that the results are robust to plausible changes in the velocity window.

In interpreting our results, we are concerned that our selection of pairs of different projected separation is somehow selecting pairs in different environments. We have previously found that the CGM emission line properties are affected by environment (Zhang et al. 2019), and therefore, an environmental difference among our various subsamples could affect what we observe here. One measure of environment that we have used previously is the number of nearby neighbors $(\mathrm{NN}$; see Zhang et al. 2019, for details).

\footnotetext{
6 The conversion factor to units between the values we present, $10^{-17} \mathrm{erg} \mathrm{cm}^{-2} \mathrm{~s}^{-1} \AA^{-1}$, and those used commonly in the literature to describe diffuse line emission, erg cm${ }^{-2} \mathrm{~s}^{-1} \operatorname{arcs}^{-2}$, is 1.7 .
} 
Table 1

The $\mathrm{H} \alpha$ and [N II] Fluxes Vs. $S_{p}$ for Different Galaxy Pair Samples

\begin{tabular}{|c|c|c|c|c|c|}
\hline \multirow{2}{*}{$\begin{array}{l}\text { Sample } \\
\text { Low Mass }\end{array}$} & \multirow{3}{*}{$\begin{array}{c}\text { Line } \\
\\
\mathrm{H} \alpha \\
{[\mathrm{N} \mathrm{II}]}\end{array}$} & \multirow{3}{*}{$\begin{array}{c}f\left(\bar{S}_{p}=32 \mathrm{kpc}\right) \\
0.033 \pm 0.005 \\
0.0069 \pm 0.0059\end{array}$} & \multicolumn{2}{|c|}{$\begin{array}{c}f\left(\bar{S}_{p}=79 \mathrm{kpc}\right) \\
\left(10^{-17} \mathrm{erg} \mathrm{cm}^{-2} \mathrm{~s}^{-1} \stackrel{\AA}{\mathrm{A}}^{-1}\right)\end{array}$} & \multirow{2}{*}{$\begin{array}{c}f\left(\bar{S}_{p}=487 \mathrm{kpc}\right) \\
0.0053 \pm 0.0014\end{array}$} \\
\hline & & & $0.0044 \pm 0.0033$ & $-0.0001 \pm 0.0023$ & \\
\hline & & & $0.0011 \pm 0.0033$ & $-0.0039 \pm 0.0024$ & $-0.0010 \pm 0.0016$ \\
\hline \multirow[t]{2}{*}{ High Mass } & $\mathrm{H} \alpha$ & $-0.0033 \pm 0.0073$ & $0.0010 \pm 0.0038$ & $-0.0013 \pm 0.0027$ & $0.0014 \pm 0.0017$ \\
\hline & {$[\mathrm{N}$ II $]$} & $0.015 \pm 0.007$ & $0.019 \pm 0.005$ & $-0.0039 \pm 0.0031$ & $0.0048 \pm 0.0018$ \\
\hline \multirow[t]{2}{*}{ Low-mass Ratio } & $\mathrm{H} \alpha$ & $0.016 \pm 0.007$ & $-0.0009 \pm 0.0035$ & $-0.0021 \pm 0.0020$ & $0.0011 \pm 0.0020$ \\
\hline & {$[\mathrm{N}$ II $]$} & $0.015 \pm 0.006$ & $0.010 \pm 0.003$ & $-0.0045 \pm 0.0022$ & $0.0012 \pm 0.0014$ \\
\hline \multirow[t]{2}{*}{ High-mass Ratio } & $\mathrm{H} \alpha$ & $0.023 \pm 0.007$ & $0.008 \pm 0.004$ & $0.0016 \pm 0.0027$ & $0.0034 \pm 0.0022$ \\
\hline & {$[\mathrm{N}$ II $]$} & $0.0025 \pm 0.0083$ & $0.007 \pm 0.004$ & $-0.0015 \pm 0.0030$ & $0.0003 \pm 0.0016$ \\
\hline
\end{tabular}

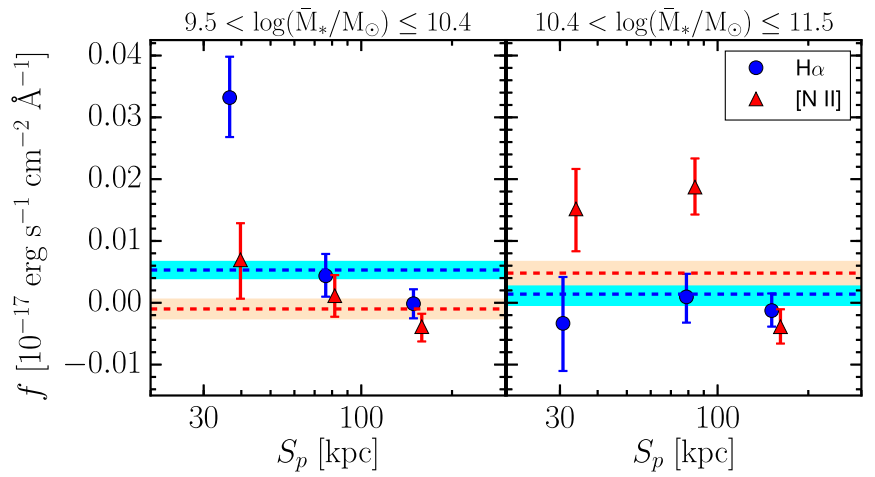

Figure 1. $\mathrm{H} \alpha$ and $[\mathrm{N} \mathrm{II]} \mathrm{emission} \mathrm{fluxes} \mathrm{as} \mathrm{a} \mathrm{function} \mathrm{of} \mathrm{projected} \mathrm{galaxy} \mathrm{pair}$ separation, $S_{p}$, for low-mass (left) and high-mass (right) pairs. For visualization, we apply slight horizontal offsets between the $\mathrm{H} \alpha$ and [N II] fluxes although they are all measured at the same $S_{p}$. The blue (red) dashed line represents the $\mathrm{H} \alpha$ ([N II]) emission flux of the control sample.

We find small, but significant differences in the mean NN value of the main and control samples, whether we consider the low- or high-mass subsamples. Furthermore, we find that the distributions of NN are statistically different with greater than 99\% confidence using a Kolmogorov-Smirnov (KS) test. Although the differences are small, they are a cause of concern. To address whether these differences are creating corresponding differences in the resulting control sample line fluxes, we create control samples that match the $\mathrm{NN}$ distribution of the comparison sample by randomly drawing an $\mathrm{NN}$-matched distribution from the full set of control sample lines of sight. We present the results in Table 2 and conclude that the differences in $\mathrm{NN}$ distributions are not responsible for the detected signal.

Similarly, we can test whether differences in the distributions of other parameters between the control and comparison samples might be affecting our result. We consider the distributions of Sérsic index, $n$, to represent morphology, $r_{p}$, $M_{*}$, and specific star formation rate, sSFR. Using a KS test to identify which parameters might present significant differences in their distributions, we find statistically significant differences only for $M_{*}$ and sSFR. We perform the same exercise as we did for NN to create control samples that match the control sample parameter distribution to that of the comparison sample. In these cases, we match to the $M_{*}$ and sSFR distributions. The results of those calculations are also presented in Table 2 . We find no significant $(>2 \sigma)$ differences in the flux values derived from the original and matched control samples.

Finally, we present the results of simultaneously matching to the distribution of sSFR, NN, and $M_{*}$. To do this, we force the 3D distribution of sSFR, NN, and $M_{*}$ of the control sample to be the same as that of the data (results in rightmost column of Table 2). This test runs the risk of dividing the sample of sight lines too finely and, by doing so, depending too much on a limited set of data. Despite this concern, our results are consistent both with our original measurements for the control samples and with the 1D distribution-matched results (again to within $2 \sigma$ ). We conclude that differences among the control and comparison samples, at least with regard to the limited parameter set explored, are not responsible for the results that we feature here.

\subsection{Emission Line Fluxes and Mass Ratio}

Our selection of galaxy pairs imposed a mass ratio limit of $1: 5$, but within that range we now define a subsample that is closer to $1: 1$, where one might expect the effect of any interaction to be stronger. In Figure 2, we present results when we divide the sample by mass ratio rather than by mean stellar mass. We find that $\mathrm{H} \alpha$ is enhanced at small galaxy pair separations in both cases $(2.5 \sigma$ and $2.8 \sigma$, low- and high-mass ratios, respectively), presumably because both mass ratio subsamples are dominated by the low-mass pairs that showed $\mathrm{H} \alpha$ enhancement at small $S_{p}$. More interestingly, we find a significant enhancement of [N II] (3.5 $\sigma$ significance when the inner two $S_{p}$ bins are combined) for pairs with mass ratios closer to $1: 1$. This enhancement is present, and even more significant among the low-mass subsample of these pairs, which overall did not show such enhancement in [N II] (Figure 1).

Again, using the $\mathrm{KS}$ test, we find slight but significant differences in the galaxy properties between main and control samples. As before, we construct parameter-matched control samples to ascertain whether those differences are in any way responsible for the results that we are finding. The fluxes derived from the parameter-matched samples are presented in Table 2 and we conclude that the differences in the control samples are not responsible for our results.

\subsection{Dependence on Geometry}

We turn to investigating the dependence of the signal on the location of the sight line relative to the pair system. We define the set of sight lines between the two galaxies of the pair to be those that lie within the smallest circle on the sky that intersects the two galaxies and refer to those as interior sight lines. The remaining sight lines outside of this circle are considered to be associated primarily with one of the two galaxies, and we refer to those as exterior sight lines.

In Figure 3 we display results obtained using only interior sight lines (left panel) and only exterior sight lines (right panel) 
Table 2

The $\mathrm{H} \alpha$ and [N II] Fluxes for the Control and Parameter-matched Control Samples

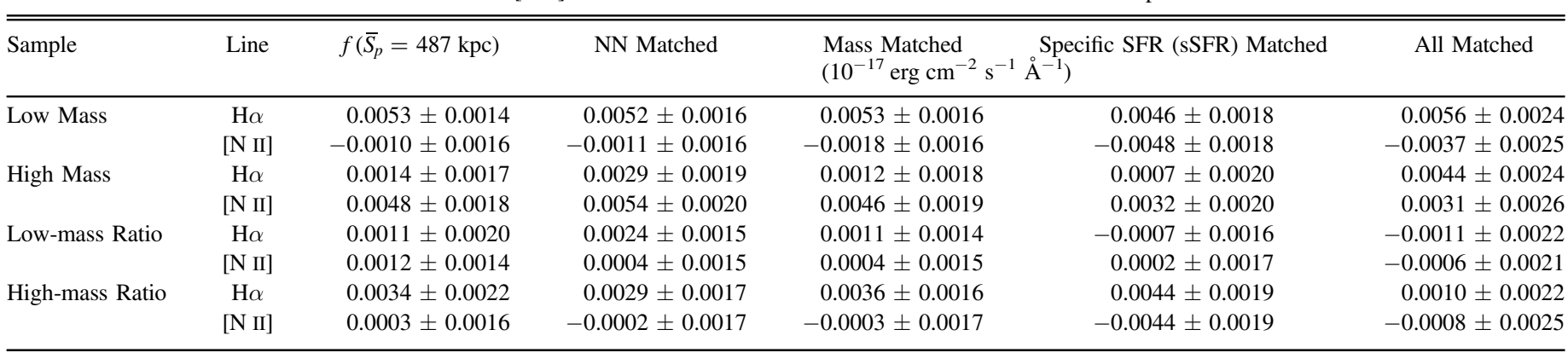

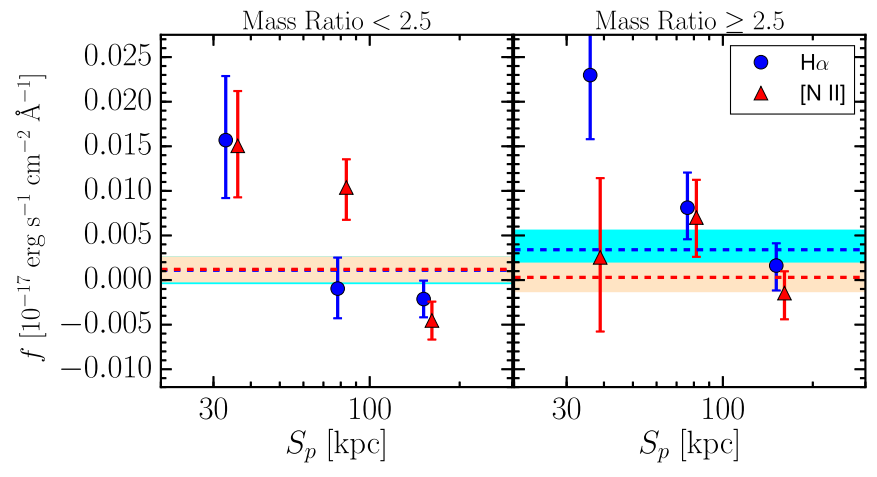

Figure 2. Left panel: emission line flux measurements for the low-mass ratio subsample as a function of galaxy pair separation. Right panel: the same measurements for the high-mass ratio subsample. The symbols are the same as in those in Figure 1.

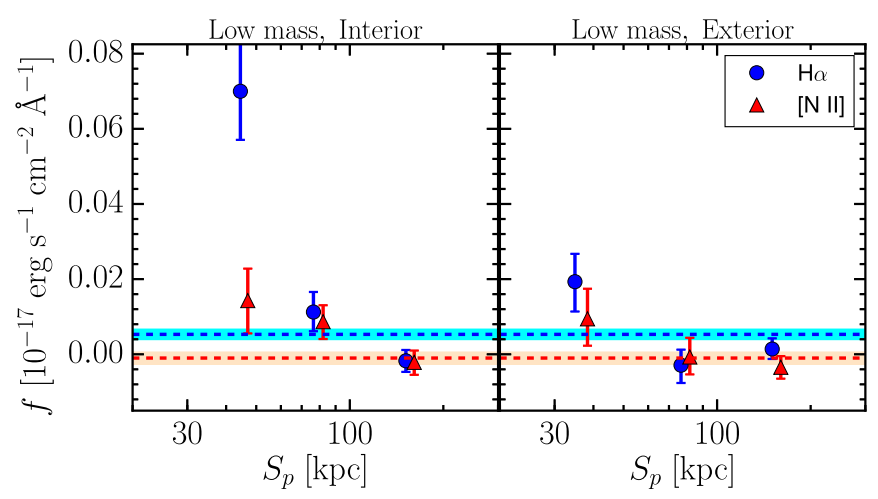

Figure 3. Left panel: emission line flux measurements for the low-mass pair sample using only interior sight lines (left panel) and only exterior sight lines (right panel) as a function of galaxy pair projected separation. The symbols are the same as in those in Figure 1.

for the low-mass pairs. The flux difference is stark and illustrates that the result shown in the left panel of Figure 1 is contributed almost entirely by interior sight lines. Because the pair galaxies are not necessarily all on first approach, we interpret this result to indicate that the elevated $\mathrm{H} \alpha$ emission is a short-lived phenomenon existing where the CGM from the two galaxies is actively interacting. We find no analogous difference among the elevated [N II] emission results when using interior and exterior sight lines for the high-mass subsample. We interpret this result to indicate that this phenomenon is long-lasting and affects the entire halo of each galaxy.

We find no significant differences in either the $\mathrm{H} \alpha$ or [N II] fluxes between the the interior and exterior sight lines for the high-mass sample. The lack of a difference in $\mathrm{H} \alpha$ is not surprising because we did not detect any $\mathrm{H} \alpha$ enhancement in the high-mass sample to begin with. However, the lack of a difference for [ $\mathrm{N} \mathrm{II]}$ is interesting and may be signaling that the elevation in [N II] flux affects the entire halo of each galaxy in the pair.

\section{Discussion}

We now briefly discuss aspects of possible interpretations of the observational results.

\subsection{Physical and Projected Galaxy Pairs}

We use cosmological simulations to estimate the fraction of physical pairs in our samples. Specifically, we use simulations that utilize halo merger trees from the Bolshoi-Planck simulation (Klypin et al. 2016; Rodríguez-Puebla et al. 2016), with halos found using the Rockstar phase-space halo finder (Behroozi et al. 2013), merger trees generated with the Consistent Trees code (Behroozi et al. 2013), and, finally, stellar masses modeled with the UniverseMachine code (Behroozi et al. 2018).

When we apply our selection criteria for the main sample, we find that $44.6 \%$ of the identified pair systems have 3D separation, $R_{3 \mathrm{D}},<200 \mathrm{kpc}$. Similarly, if we limit our selection to pairs with $S_{p}<50 \mathrm{kpc}$, we find that $43.6 \%$ have $R_{3 \mathrm{D}}<50 \mathrm{kpc}$. Both of these results suggest that our measured line emission enhancements are likely underestimated, perhaps by as a much as a factor of two, due to significant contamination by wider separation system.

\subsection{Outer Region Star Formation}

In the low-mass pair sample, we find elevated $\mathrm{H} \alpha$ fluxes in the innermost bin. This may be the result of changing CGM conditions in these interacting systems. For example, the interaction might have increased the density of the emitting gas and therefore increased the emission, which depends on density squared. Simulations, which are used to inform the interpretation of observed absorption lines, find significantly higher $\mathrm{H}$, $\mathrm{C}$, and $\mathrm{O}$ covering fractions in the vicinity of merging galaxies compared to those of isolated galaxies (Hani et al. 2018), although model-dependent ionization effects can lead to decreases in the covering fraction of associated, commonly observed ions (HI, C IV, O VI). This complimentary result suggests a higher level of emission from the CGM in galaxy mergers, as we have found in the current study.

On the other hand, interactions are often considered to give rise to increased star formation. This may even be true in the 
outer disks of galaxies. One spectacular example of outer disk star formation, M83 (Thilker et al. 2005), also appears to have interacted recently (Malin \& Hadley 1997). The outer disk starforming regions, at least in M83, extend out to several tens of $\mathrm{kpc}$. In a sample of nearby $\mathrm{H}$ I-selected galaxies, Werk et al. 2010 find that $\sim 10 \%$ show outlying $\mathrm{HII}$ regions in $\mathrm{H} \alpha$ emission beyond $2 \times$ the 25 th magnitude $R$-band isophotal radius, often well beyond $20 \mathrm{kpc}$. Although our stacking methodology rejects lines of sight with anomalously high $\mathrm{H} \alpha$ fluxes, some lines of sight through the diffuse outer disks of systems like M83 could perhaps be responsible for the observed excess. Imaging of the emission lines in pair systems will allow us to determine if the excess comes from highly localized star formation regions or is due to a more general halo phenomenon.

\subsection{Signature of Shocks}

We have previously exploited line ratios to help us understand the nature of excitation mechanism of the lines that we are observing (Zhang et al. 2018b). Here, we are particularly interested in the role that shocks may be playing when the galaxies interact. As discussed by Rich et al. (2010) and Farage et al. (2010), shock models, for velocities $>200 \mathrm{~km} \mathrm{~s}^{-1}$, predict $\log ([\mathrm{N} \mathrm{II}] 6583 / \mathrm{H} \alpha)>0.2$ and $\log$ $([\mathrm{O} \mathrm{III}] / \mathrm{H} \beta)<0$. Of particular interest is that we find that $\log ([\mathrm{N} \mathrm{II}] 6583) / \mathrm{H} \alpha)$ increases in the interacting systems, and increases into a range of values consistent with shock excitation. The lower limit of $\log ([\mathrm{N} \mathrm{II}] 6583) / \mathrm{H} \alpha)$ that we obtain for the high-mass subsample is 0.65 . This value is a lower limit because we calculate it using the upper bound on the $\mathrm{H} \alpha$ flux (our measurement for the $\mathrm{H} \alpha$ flux is formally negative, even though it is consistent with small positive values given the uncertainties). Because this value of the line ratio is well above the threshold of 0.2 , we suggest that we may be viewing CGM shocks in interacting galaxy pairs. Furthermore, we note that the increase in [N II] is most noticeable when the galaxies are comparable in mass (Figure 2), and so shocks presumably have comparable effects on the CGM surrounding both members.

This is not the first indication of shocks in the CGM of interacting galaxies. For example, Yoshida et al. (2016) noted that the correspondence between the $\mathrm{H} \alpha$-emitting nebula surrounding the starburst/merging galaxy NGC 6240 and the $\mathrm{X}$-ray $(0.7-1.1 \mathrm{keV})$ emitting hot gas (Nardini et al. 2013) suggests shock-heating as a way to excite both phases of the gas. Unfortunately, there are complicating factors in the interpretation of our result, and sophisticated modeling will ultimately be needed. For example, $\log ([\mathrm{N} \mathrm{II}] 6583 / \mathrm{H} \alpha)$ is known to also be a metallicity indicator (Pettini \& Pagel 2004). The simulations of Hani et al. (2018) indeed find that galaxy major mergers increase the CGM metallicity by $0.2-0.3$ dex immediately following the interaction. As such, some of the variation that we observe in this line ratio could also be due to either the introduction of low-metallicity gas at small radii or the dredging up of higher-metallicity gas from inner radii out into the halo.

\section{Summary}

We present measurements of $\mathrm{H} \alpha$ and [N II] emission line flux as a function of projected distance for galaxy pairs. We define the sample by requiring that the galaxy pair projected distance between the two galaxies be less than $200 \mathrm{kpc}$, that the recessional velocity difference be less than $1000 \mathrm{~km} \mathrm{~s}^{-1}$, and that the mass ratio be less than 1:5. Meanwhile, we construct a control sample where the only difference is that the galaxy pair projected distance range is from 400 to $600 \mathrm{kpc}$. We measure the $\mathrm{H} \alpha$ and [N II] emission line flux within projected separations of $10<r_{p} / \mathrm{kpc}<50$ around either galaxy of the pair system.

We identify a variety of differences in the CGM properties of galaxy pairs in our main and control samples. First, we find that for lower-mass pairs (mean stellar mass of $10^{10.03} M_{\odot}$ ), there is a significant $(>4.7 \sigma)$ enhancement in the $\mathrm{H} \alpha$ emission line flux, relative to that in control sample, at small separations (mean projected separation, $\bar{S}_{p},=32 \mathrm{kpc}$ ). At other separations less than $200 \mathrm{kpc}$ the data and control sample are statistically consistent for both $\mathrm{H} \alpha$ and [N II]. Second, among high-mass pairs (mean stellar mass of $10^{10.68} M_{\odot}$ ), [N II] emission line flux is significantly $(>2.5 \sigma)$ enhanced for $S_{p}<100 \mathrm{kpc}$. $\mathrm{H} \alpha$ emission flux is consistent with the control value at all radii.

In results that further constrain possible interpretations, we also find that the mass ratio of the pair plays a role. We detect a statistically significant $(3.5 \sigma)$ enhancement of the [N II] emission line flux for the half of our pair sample with mass ratios closer to $1: 1$ and no such enhancement among the higher mass ratio pairs. Furthermore, we find that the bulk of the $\mathrm{H} \alpha$ flux enhancement at low projected separations for the low-mass pair sample comes from lines of sight located between the two galaxies. We interpret this result to indicate that the elevated $\mathrm{H} \alpha$ emission is a short-lived phenomenon existing where the CGM from the two galaxies is actively interacting. We find no analogous difference for the high-mass subsample, which we interpret to indicate that in this case the phenomenon is longlasting and affects the entire halo of each galaxy.

The rise in $\mathrm{H} \alpha$ could be attributed to a variety of factors, but we attribute the increase of [N II] to shocks. The preliminary indication is that the manifestation of shocks is strongest in the more massive systems and in those pairs where the mass ratio is closer to $1: 1$. We conclude that interactions begin to grossly affect the CGM at separations $\lesssim 100 \mathrm{kpc}$, which then, presumably, affects how that gas interacts with the central galaxy. Emission line maps of such systems would be of tremendous value in assessing interaction between the circumgalactic media of the interacting galaxies.

D.Z. and H.Z. acknowledge financial support from NSF grant AST-1311326. T. Fang is supported by the National Key R\&D Program of China No. 2017YFA0402600, and NSFC grants No. 11525312, 11890692. J.W. acknowledges support from a 2018 Sloan Foundation Fellowship. This work is supported by the 973 Program (Nos. 2015CB857002) and national science foundation of China (grant Nos. 11833005, 11890692, 11621303). We thank the referee for their constructive suggestions. We also thank the support of the Key Laboratory for Particle Physics, Astrophysics and Cosmology, Ministry of Education. The authors gratefully acknowledge the SDSS III team for providing a valuable resource to the community. Funding for SDSS III has been provided by the Alfred P. Sloan Foundation, the Participating I institutions, the National Science Foundation, and the U.S. Department of Energy Office of Science. The SDSS III website is http://www.sdss3.org/. 
SDSS III is managed by the Astrophysical Research Consortium for the Participating Institutions of the SDSS III Collaboration including the University of Arizona, the Brazilian Participation Group, Brookhaven National Laboratory, Carnegie Mellon University, University of Florida, the French Participation Group, the German Participation Group, Harvard University, the Instituto de Astrofisica de Canarias, the Michigan State/Notre Dame/JINA Participation Group, Johns Hopkins University, Lawrence Berkeley National Laboratory, Max Planck Institute for Astrophysics, Max Planck Institute for Extraterrestrial Physics, New Mexico State University, New York University, Ohio State University, Pennsylvania State University, University of Portsmouth, Princeton University, the Spanish Participation Group, University of Tokyo, University of Utah, Vanderbilt University, University of Virginia, University of Washington, and Yale University.

\section{ORCID iDs}

Huanian Zhang

(张华 年) (10 https://orcid.org/0000-0002-0123-9246

Taotao Fang

(方陶 陶) (10 https://orcid.org/0000-0002-2853-3808

Dennis Zaritsky (ib https://orcid.org/0000-0002-5177-727X

Jessica Werk (i) https://orcid.org/0000-0002-0355-0134

Xiaohu Yang

(杨小 虎) (1D https://orcid.org/0000-0003-3997-4606

\section{References}

Abazajian, K. N., Adelman-McCarthy, J. K., Agüeros, M. A., et al. 2009, ApJS, 182, 543

Alam, S., Albareti, F. D., Allende Prieto, C., et al. 2015, ApJS, 219, 12

Bahcall, J. N., \& Spitzer, L. J. 1969, ApJL, 156, L63

Barnes, J. E., \& Hernquist, L. 1996, ApJ, 471, 115

Behroozi, P., Wechsler, R., Hearin, A., \& Conroy, C. 2018, MNRAS, 488, 3143

Behroozi, P. S., Wechsler, R. H., \& Wu, H.-Y. 2013, ApJ, 762, 109

Behroozi, P. S., Wechsler, R. H., Wu, H.-Y., et al. 2013, ApJ, 763, 18

Boksenberg, A., \& Sargent, W. L. W. 1978, ApJ, 220, 42

Bordoloi, R., Lilly, S. J., Knobel, C., et al. 2011, ApJ, 743, 10

Brinchmann, J., Charlot, S., White, S. D. M., et al. 2004, MNRAS, 351, 1151

Cai, Z., Fan, X., Yang, Y., et al. 2017, ApJ, 837, 71

Chen, H.-W. 2017a, in Outskirts of Galaxies, Astrophysics and Space Science Library, Vol. 434, ed. J. H. Knapen, J. C. Lee, \& A. Gil de Paz (Berlin: Springer), 291

Chen, H.-W. 2017b, in Gas Accretion onto Galaxies, Astrophysics and Space Science Library, Vol. 430, ed. A. Fox \& R. Davé (Berlin: Springer), 167

Chen, H.-W., Boettcher, E., Johnson, S. D., et al. 2019, ApJL, 878, L33

Croft, R. A. C., Miralda-Escudé, J., Zheng, Z., et al. 2016, MNRAS, 457, 3541

Croft, R. A. C., Miralda-Escudé, J., Zheng, Z., Blomqvist, M., \& Pieri, M. 2018, MNRAS, 481, 1320

Ellison, S. L., Mendel, J. T., Patton, D. R., \& Scudder, J. M. 2013, MNRAS, 435,3627
Farage, C. L., McGregor, P. J., Dopita, M. A., \& Bicknell, G. V. 2010, ApJ, 724,267

Gallazzi, A., Charlot, S., Brinchmann, J., White, S. D. M., \& Tremonti, C. A. 2005, MNRAS, 362, 41

Hani, M. H., Sparre, M., Ellison, S. L., Torrey, P., \& Vogelsberger, M. 2018, MNRAS, 475, 1160

Hopkins, P. F., \& Hernquist, L. 2006, ApJS, 166, 1

Johnson, S. D., Chen, H.-W., Straka, L. A., et al. 2018, ApJL, 869, L1

Joshi, R., Srianand, R., Petitjean, P., \& Noterdaeme, P. 2018, MNRAS, 476, 210

Kauffmann, G., Heckman, T. M., White, S. D. M., et al. 2003a, MNRAS, 341,54

Kauffmann, G., Heckman, T. M., White, S. D. M., et al. 2003b, MNRAS, 341, 33

Kereš, D., Katz, N., Weinberg, D. H., \& Davé, R. 2005, MNRAS, 363, 2

Kewley, L. J., Geller, M. J., \& Barton, E. J. 2006, AJ, 131, 2004

Kewley, L. J., Rupke, D., Zahid, H. J., Geller, M. J., \& Barton, E. J. 2010 , ApJL, 721, L48

Klypin, A., Yepes, G., Gottlöber, S., Prada, F., \& Heß, S. 2016, MNRAS, 457,4340

Lan, T.-W., \& Mo, H. 2018, ApJ, 866, 36

Malin, D., \& Hadley, B. 1997, PASA, 14, 52

Ménard, B., Wild, V., Nestor, D., et al. 2011, MNRAS, 417, 801

Nardini, E., Wang, J., Fabbiano, G., et al. 2013, ApJ, 765, 141

Pettini, M., \& Pagel, B. E. J. 2004, MNRAS, 348, L59

Planck Collaboration et al. 2018, arXiv:1807.06205

Prochaska, J. X., Werk, J. K., Worseck, G., Tripp, T. M., Tumlinson, J., et al. 2017, ApJ, 837, 169

Rampazzo, R., Plana, H., Amram, P., et al. 2005, MNRAS, 356, 1177

Rich, J. A., Dopita, M. A., Kewley, L. J., \& Rupke, D. S. N. 2010, ApJ, 721,505

Riess, A. G., Casertano, S., Yuan, W., et al. 2018, ApJ, 861, 126

Rodríguez-Puebla, A., Behroozi, P., Primack, J., et al. 2016, MNRAS, 462,893

Simard, L., Mendel, J. T., Patton, D. R., Ellison, S. L., \& McConnachie, A. W. 2011, ApJs, 196, 11

Spitzer, L., Jr. 1956, ApJ, 124, 20

Springel, V., Di Matteo, T., \& Hernquist, L. 2005, ApJL, 620, L79

Steidel, C. C., Erb, D. K., Shapley, A. E., et al. 2010, ApJ, 717, 289

Thilker, D. A., Bianchi, L., Boissier, S., et al. 2005, ApJL, 619, L79

Toomre, A., \& Toomre, J. 1972, ApJ, 178, 623

Tumlinson, J., Peeples, M. S., \& Werk, J. K. 2017, ARA\&A, 55, 389

Tytler, D. 1982, Natur, 298, 427

Werk, J. K., Prochaska, J. X., Cantalupo, S., et al. 2016, ApJ, 833, 54

Werk, J. K., Prochaska, J. X., Tumlinson, J., et al. 2014, ApJ, 792, 8

Werk, J. K., Putman, M.E., Meurer, G.R., et al. 2010, AJ, 139, 279

Yoshida, M., Yagi, M., Ohyama, Y., et al. 2016, ApJ, 820, 48

Zabludoff, A. I., Zaritsky, D., Lin, H., et al. 1996, ApJ, 466, 104

Zahedy, F. S., Chen, H.-W., Johnson, S. D., et al. 2019, MNRAS, 484, 2257

Zahedy, F. S., Chen, H.-W., Rauch, M., \& Zabludoff, A. 2017, ApJL, 846, L29

Zhang, H., Yang, X., Zaritsky, D., Behroozi, P., \& Werk, J. 2020, arXiv:1911. 02032

Zhang, H., Zaritsky, D., \& Behroozi, P. 2018a, ApJ, 861, 34

Zhang, H., Zaritsky, D., Behroozi, P., \& Werk, J. 2019, ApJ, 880, 28

Zhang, H., Zaritsky, D., Werk, J., \& Behroozi, P. 2018b, ApJ, 866, L4

Zhang, H., Zaritsky, D., Zhu, G., Ménard, B., \& Hogg, D. W. 2016, ApJ, 833,276

Zhu, G., \& Ménard, B. 2013a, ApJ, 773, 16

Zhu, G., \& Ménard, B. 2013b, ApJ, 770, 130 